\title{
Prospects for improved quantitative electron diffraction.
}

\author{
Richard Beanland \\ University of Warwick, Coventry, United Kingdom \\ r.beanland@warwick.ac.uk
}

Crystal structure solution and refinement using three-dimensional electron diffraction (3DED) is on the cusp of becoming a widelyused technique and it seems certain that it will be used for many applications in the near future. Nevertheless, in comparison with Xray diffraction there is still poor agreement between calculated and observed intensities for 3DED data. Since it is very well-known that the kinematical (single-scattering) theory fails to describe most electron scattering, these discrepancies have been usually explained using the catch-all excuse of 'dynamical (multiple scattering) effects'.

However, when dynamical electron diffraction effects are measured carefully, and compared with standard electronscattering theory, agreement between experiment and simulation is actually very good. The dynamical scattering effects contain phase information that is extremely sensitive to the details of the inner potential of the material and can be used to determine atomic coordinates to a precision of $\sim 0.1 \mathrm{pm}$. Examples are given using using 'digital' large-angle convergent beam electron diffraction (D-LACBED).

In order to progress in the accuracy of 3DED work we therefore need to pay attention to the experimental parameters which are specific to the method. These parameters combine with dynamical effects to change diffracted intensities and include incident beam convergence, the effect of intermediate lenses between the crystal and detector, crystal orientation, shape, defectivity, mosaicity, inelastic scattering and beam damage. Improvements in 3DED will depend on the ability to measurethese parameters and refine them using measured intensities.

Keywords: Electron diffraction, dynamical diffraction, 3DED, D-LACBED 